Essers, G., Dulmen, S. van, Es, J. van, Weel, C. van, Vleuten, C. van der, Kramer, A. Context factors in consultations of general practitioner trainees and their impact on communication assessment in the authentic setting. Patient Education and Counseling: 2013, 93(3), 567-572

nivel

\begin{tabular}{|l|l|}
$\begin{array}{l}\text { Postprint } \\
\text { Version }\end{array}$ & 1.0 \\
\hline Journal website & http://www.sciencedirect.com/science/article/pii/S0738399113003510 \\
\hline Pubmed link & $\underline{\text { http://www.ncbi.nlm.nih.gov/pubmed/24041713 }}$ \\
\hline DOI & 10.1016/j.pec.2013.08.024
\end{tabular}

This is a NIVEL certified Post Print, more info at http://www.nivel.eu

\title{
Context factors in consultations of general practitioner trainees and their impact on communication assessment in the authentic setting
}

\author{
GEURT ESSERS $^{\mathrm{A},}$ SANDRA VAN DULMEN ${ }^{\mathrm{A}, \mathrm{B}, \mathrm{C}}$, JUDY VAN ES ${ }^{\mathrm{D}}$, CHRIS VAN WEEL ${ }^{\mathrm{A}, \mathrm{E}}$, CEES \\ VAN DER VLEUTEN $^{A, F, G, H}$, ANNEKE KRAMER ${ }^{A}$ \\ ${ }^{a}$ Department of Primary \& Community Care, Radboud University Nijmegen Medical Centre, \\ Nijmegen, The Netherlands

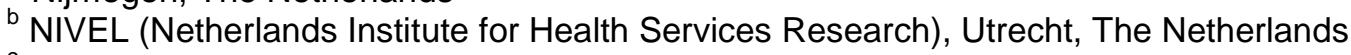 \\ ${ }^{c}$ Department of Health Science, Buskerud University College, Drammen, Norway \\ ${ }^{\mathrm{d}}$ Department of General Practice, Academic Medical Centre, University of Amsterdam, \\ Amsterdam, The Netherlands \\ ${ }^{\mathrm{e}}$ Australian Primary Health Care Research Institute, Australian National University, \\ Canberra, Australia \\ ${ }^{\dagger}$ Department of Educational Development and Research, Maastricht University, Maastricht, \\ The Netherlands \\ ${ }^{g}$ Northumbria University, Newcastle upon Tyne, United Kingdom \\ ${ }^{\mathrm{h}}$ Copenhagen University, Copenhagen, Denmark
}

\section{Abstract \\ Objective}

Acquiring adequate communication skills is an essential part of general practice (GP) specialty training. In assessing trainee proficiency, the context in which trainees communicate is usually not taken into account. The present paper aims to explore what context factors can be found in regular GP trainee consultations and how these influence their communication performance.

Methods

In a randomly selected sample of 44 videotaped, real-life GP trainee consultations, we searched for context factors previously identified in GP consultations and explored how trainee ratings change if context factors are taken into account. Trainee performance was rated twice using the MAASGlobal, first without and then with incorporating context factors. Item score differences were calculated using a paired samples t-test and effect sizes were computed.

Results

All previously identified context factors were again observed in GP trainee consultations. In communication assessment scores, we found a significant 
Essers, G., Dulmen, S. van, Es, J. van, Weel, C. van, Vleuten, C. van der, Kramer, A. Context factors in consultations of general practitioner trainees and their impact on communication assessment in the authentic setting. Patient Education and Counseling: 2013, 93(3), 567-572

difference in 5 out of 13 MAAS-Global items, mostly in a positive direction. The effect size was moderate (0.57).

Conclusions

GP trainee communication is influenced by contextual factors; they seem to adapt to context in a professional way.

Practice implications

GP specialty training needs to focus on a context-specific application of communication skills. Communication raters need to be taught how to incorporate context factors into their assessments.

\section{INTRODUCTION}

In postgraduate specialty training, working in daily practice is considered to create an ideal and essential learning environment for acquiring the necessary professional expertise [1]. During the GP specialty training, learning adequate communication skills is part of the programme and supported by off-the-job training. For the most part, the trainee's learning process is guided by experiences in daily practice. There are indications, however, that the growth of communication skills of GP trainees is limited [2], [3], [4] and [5] although two recent studies show that some improvement can be found [6] and [7]. This limited growth may be attributed to several causes, such as transfer problems [8] and [9] or a communication training programme that is not sufficiently aligned with the context of daily practice [10] and [11] and the personal learning needs of the participants [12], [13], [14], [15] and [16]. However, it may also be due to the way communication performance is assessed [17], [18] and [19].

Communication levels are usually assessed by rating the performance against predefined generic communication skills standards [11], [20] and [21]. However, communication is a goal-oriented activity [22] and context-dependent [10], [11], [17], [22], [23], [24], [25], [26], [27] and [28]. The assumption that workplace-based assessment of communication skills can be done in a non-specific, contextindependent way may therefore be unjustified [17], [19], [22] and [29]. If we want to acknowledge context influences on communication, assessment of communication performance in daily practice consultations should be done in a context-specific way. In a previous study, we found indications that GP communication performance scores are higher if context factors are taken into account [30]. The influence of context-specific assessment on GP trainee performance scores has not been studied yet. We know that there are considerable differences between the patient mixes of trainers and trainees during GP specialty training [31]. Moreover, patients tend to present less chronic or emotional problems in trainee consultations [32]. Therefore, it would be useful first to verify if the same context factors can be found in GP trainee consultations as were found previously in GP consultations [33].

Furthermore, it is unclear to what extent differences in context factors affect the assessment of GP trainee communication. Trainees possibly encounter fewer context factors that have shown to influence communication in GP consultations or are less skilful in dealing with context factors, indicating that GP trainees may have things to learn in handling context factors in a consultation. Therefore, in this exploratory study, our first research question is: What context factors can be identified in GP 
Essers, G., Dulmen, S. van, Es, J. van, Weel, C. van, Vleuten, C. van der, Kramer, A. Context factors in consultations of general practitioner trainees and their impact on communication assessment in the authentic setting. Patient Education and Counseling: 2013, 93(3), 567-572

trainee consultations? The second research question is: How do communication performance scores of GP trainees change if context factors are taken into account? The results were compared to those of a reference group of experienced GPs from a previous study [30].

\section{METHODS}

\subsection{Data set and sample size}

To answer both of our questions, we used a set of real-life videotaped GP traineepatient consultations, taken from a database of 467 videotaped consultations which were recorded as part of a video-observation study performed by AMC Amsterdam [34]. The GP trainees were in their first year of the GP specialty training, doing their own surgeries. The consultations were recorded for research purposes at 9 months in their first GP internship and were not part of a formal, summative assessment in the training programme.

A sample of 44 consultations would provide enough power to establish a minimal relevant difference between the two ratings of $0.45(\alpha=0.05, \beta=0.10)$ on item scores [6]. Therefore, 22 out of 29 GP trainees who had provided digital recordings, were randomly selected. From each of these trainees we selected two (the 4th and 5th) consultations. We excluded the first three consultations in order to minimise the stage-effect for the trainee having to get used to the video recording.

\subsection{Procedure}

To answer the first research question, each consultation of the sample with 44 consultations was viewed by one rater (BA), who examined the presence of context factors using the list resulting from our previous study [30] (see Appendix 1 for definitions, operationalisation and examples of previously identified context factors). To answer the second research question, another rater (GE) rated the same sample of 44 consultations twice using the same rating instrument (the MAAS-Global) [35]. The rating of the GP trainee communication performance was purposefully done both times by the same rater (GE) to exclude noise produced by heterogeneity of raters [36]. Intra-rater reliability had been found good in the previous study [33]. The first rating was performed in the standard way, following the guidelines of the MAAS-Global Manual. In the second rating, context factors were explicitly taken into account (see Fig. 1). For the incorporation of context factors into the rating process, we used a context-specific rating protocol, developed in our previous study, in addition to the MAAS-Global Manual [30]. In between standard and contextspecific rating, a second rater (BA), a GP and an experienced faculty member, observed and rated the consultations for the presence of context factors, by using the list of previously identified context factors shown in Table 1. Prior to the contextspecific rating, GE received a data sheet from BA on the presence of context factors in each of the consultations. Differences on the identification of context factors that arose during the second rating were discussed between GE and BA until agreement was reached. Subsequently, GE noted the presence of the context factors on the MAAS-Global sheet for each consultation and indicated which of the new rating rules could be applicable in that consultation. After this, the GP communication performance was rated the second time using the adapted MAAS-Global protocol. 
Essers, G., Dulmen, S. van, Es, J. van, Weel, C. van, Vleuten, C. van der, Kramer, A. Context factors in consultations of general practitioner trainees and their impact on communication assessment in the authentic setting. Patient Education and Counseling: 2013, 93(3), 567-572

The study was carried out between July 2011 and September 2012. The time lapse between first (standard) and second (context-specific) rating was 13 months.

\subsection{Observational framework}

The MAAS-Global is a validated communication assessment instrument, which serves as a guideline for patient-centred and task-oriented medical communication [20] and [37]. It is widely used in undergraduate medical and GP specialty training in the Netherlands [38] and [39]. The MAAS-Global consists of 13 generic communication items that can be rated on a 7-point Likert scale, ranging from 0 ('absent') to 6 ('excellent'). Two items can also be scored 'Not applicable'. Each item has three or four sub-items that indicate criterion behaviour (Appendix 2). The MAAS-Global Manual offers guidelines to rate communication skills, and acknowledges that context factors play a role but leaves implicit how to incorporate contextual influences.

\section{[FIGURE 1][TABLE 1]}

The context-specific rating protocol accounts for the absence of certain communication skills as a result of the presence of one or more context factors and at the same time keeps the existing rating rules of the MAAS-Global Manual intact.

Appendix 3 presents the protocol and the ascertained relationships. In order to stay in line with the MAAS-Global rating rules, the central assumptions in the protocol were:

- If a sub-item is justifiably absent as a result of the presence of one or more context factors, it should not weigh on the item scores.

- If an item is justifiably absent due to the presence of one or more context factors, it should be scored 'Not applicable'.

In order to perform the context-specific rating, on the MAAS-Global scoring form for each consultation, the presence of context factors was noted. Having established the context factors in the consultations, the context-specific rating protocol could be applied.

\subsection{Analysis}

In order to establish the presence of context factors in the GP trainee consultations, the rater used the list of context factors that were identified in a previous study, shown in Table 1[33]. Moreover, the rater looked for new context factors in the GP trainee setting. Findings were compared to those of a reference group of experienced GPs from a previous study [30].

To analyse the influence of context factors on trainee rating, we calculated the number of applicable MAAS-Global items per consultation, with and without accounting for context factors, as this number is used as the denominator to determine the overall score on the MAAS-Global [6], [36], [37] and [40]. The effect of the context-specific rating was tested by calculating the significance of the change in scores, using a paired $t$-test for repeated measurement in the same sample (SPSS 20.0). To determine the relevance of the difference between the two ratings, the effect size was calculated for the difference between the individual MAAS-Global item scores and for the difference between the total average scores per consultation, divided by their pooled initial SDs; a $d$ of 0.2 was considered a small effect, a $d$ of 0.5 as a moderate effect, and a $d$ of 0.8 as a large effect [36], [41] 
Essers, G., Dulmen, S. van, Es, J. van, Weel, C. van, Vleuten, C. van der, Kramer, A. Context factors in consultations of general practitioner trainees and their impact on communication assessment in the authentic setting. Patient Education and Counseling: 2013, 93(3), 567-572

and [42]. Mean items scores and total average scores as well as effect sizes were compared to those of the reference group of experienced GPs [30].

\section{[TABLE 2]}

\subsection{Ethical regulations}

The study was performed according to Dutch privacy legislation. The privacy regulation was approved by the Dutch Data Protection Authority. All participating GP trainees and patients gave informed consent for recording the consultation. According to Dutch legislation, approval by a medical ethics committee was not required for this observational study.

\section{RESULTS}

\subsection{Sample characteristics}

The 22 GP trainees mean age was 29.5 (SD: 4.2) and 17.4\% was male. In the overall GP trainee population, mean age in 2009 was 31.3 and $28.6 \%$ was male. The 20 GPs in the sample, that we compared the trainees with on the presence and influence of context factors, were comparable in gender, age, and practice type to the Dutch GP population (35\% female GPs, mean age 49 years (SD: 6.4) vs. 51 years (SD: 5.9 )), although urban practices were somewhat overrepresented [43].

\subsection{Presence of context factors}

In the GP trainee consultations, we actually found all 19 context factors that were previously identified in GP consultations and no new ones were identified (Table 1). There was no need to change the categorisation of the context factors (doctor-related, patient-related, and consultation-related factors).

Table 1 lists the context factors found in the GP trainee and GP consultations, and their frequencies. Doctor-related factors show the largest frequency differences: GPs seemed to know the patients more often than GP trainees (29 out of 40 vs. 11 out of $25)$, as well as their patients' way of communicating (29/40 vs. 12/44). They also seemed more informed about their medical histories (35/40 vs. 21/44) and were more experienced. Except for small differences in specific non-verbal behaviour (1/40 vs.

9/44) and in patients' familiarity with the physical examination (23/40 vs. 28/44), there were no frequency differences observed in the patient-related context factors and few such differences observed in consultation related context factors. GP trainees seemed to encounter slightly more single consultations (26/44 vs. 23/40), and more first consultations in a series (10/44 vs. 4/40) but fewer follow-up consultations (9/44 vs. 11/40). Furthermore, GPs more often saw more than one person in their consultations (12/40 vs. 8/44).

\subsection{Influence of context factors on assessment outcomes}

For GP communication assessment scores, we found a significant difference in 5 out of 13 MAAS-Global items between scores with or without incorporating context factors (in Opening, Physical Examination, Management, Summarising and Structuring) (see Table 2). The scores in almost all items (except for Closure) went up. Effect sizes of the changes in scores are small, in one item moderate (Structuring), and in two items large (Introduction and Physical Examination). The effect size of the mean overall scores was moderate (0.57). 
Essers, G., Dulmen, S. van, Es, J. van, Weel, C. van, Vleuten, C. van der, Kramer, A. Context factors in consultations of general practitioner trainees and their impact on communication assessment in the authentic setting. Patient Education and Counseling: 2013, 93(3), 567-572

\section{DISCUSSION AND CONCLUSIONS}

\subsection{Discussion}

Despite differences in patient mixes and the fact that patients tend to present less with chronic or emotional problems in trainee vs. GP consultations [31] and [32], we found the same context factors in GP and GP-trainee consultations. Moreover, no new ones were identified. This finding is striking as it indicates that context factors may be just as relevant to GP trainee consultations as they are to GP consultations although their frequencies may differ. Obviously, the largest frequency differences were seen in the doctor-related context factors; as a consequence of having worked in the same practice for years, GPs seem to know their patients better in the medical, psychological, as well as social sense. Furthermore, GPs were unsurprisingly observed to be more experienced than GP trainees (34/40 vs. 4/44). The sample we used being relatively small, we cannot attach too much relevance to the frequencies we found. To assess frequency differences, the study should be replicated with larger samples.

Theoretically, being seen by a GP trainee can be considered a context factor of its own: patients will need to have more patience, as the trainee is less sure of himself and his judgement, has less internalised illness scripts, demonstrates more explicit summarisations and, all in all, is slower in his consultations [32]. Moreover, different communication patterns identified by conversation analysis showed that experienced GPs are better at responding to the information provided by their patients, and adapt and structure the consultation accordingly. Experienced GPs tend to less hang on to a fixed structure. In contrast, GP trainees appear to focus on the structure of the consultation and only bring up new topics when they feel ready to do so [44].

However, 'being a trainee' does not meet the criteria we set for a context factor to be

identified as such. Therefore, we do not consider 'being a trainee' a context factor for communication assessment.

Incorporating context factors in assessment has a smaller, but still moderate, influence on performance outcomes in GP trainees vs. GPs (Table 2). In GP assessment, we found significant differences in 11 out of 13 item scores, whereas in GP trainee consultations, these were found in only 5 items. Furthermore, effect sizes of the changes of GP trainee scores are smaller than those in GP scores, except for the items Introduction and Physical Examination (see Table 2). This more moderate effect in trainee consultations is logical firstly from a chance perspective, as there are more context factors present in GP consultations that can produce justifiable differences in assessment than there are in GP trainee consultations (255 vs. 196). Moreover, it is logical from the difference in doctor-related context factors: GPs know their patients better and have more experience than GP trainees. The larger effect sizes in trainee consultations on the items introduction and physical examination may be explained by the fact that trainees tend to spend more time on these aspects than experienced GPs [45]. Although there was no need for it in the specific context, the trainees tended to communicate on these aspects and were valued for it. Apart from that, the small sample size may have caused this somewhat unexpected effect.

In some ways, GP trainee communication seems more 'by the book' than that of GPs, which is reflected by the higher scores of GP trainees in the item Request for help; in 
Essers, G., Dulmen, S. van, Es, J. van, Weel, C. van, Vleuten, C. van der, Kramer, A. Context factors in consultations of general practitioner trainees and their impact on communication assessment in the authentic setting. Patient Education and Counseling: 2013, 93(3), 567-572

the GP trainee's off-the-job training, this item is emphasised over and over again as an important aspect of the doctor-patient encounter. Although there is a possible pitfall, an experienced GP who knows his patients, when consulting with a patient, may more often anticipate where the consultation is going rather than explicitly explore the patient's current request for help. Furthermore, experienced GPs rely on contextual information more often in their diagnostic tasks, which will influence communication patterns [45]. Also with respect to structuring, a higher effect was to be expected in GP consultations due to their experience [44]. Nevertheless, the results also suggest that GP trainee communication is influenced by contextual factors too and that, in communicating with patients in everyday, authentic consultations, they will develop their experience and deal with these context factors in a professional way. This would suggest that incorporating context factors in communication is an effect of accumulating professional expertise.

\subsubsection{Strengths and limitations}

This study was based on 44 GP trainee consultations. Therefore, the results should be considered with care; we have found indications of the direction that context-specific assessment may take in GP trainee consultations. Also context factor frequencies found in this study cannot be generalised, as the sample is not sufficiently large for this; the results only represent the consultations in the sample. Furthermore, as this study aimed to explore the effect on item scores, the results cannot be generalised to all GP trainee communication scores.

In assessing the presence of context factors, some subjectivity is necessarily involved. For our study, substantial inter-rater agreement between two raters was found in determining the presence of context factors in GP consultations [33]. As is shown in other studies on assessment of clinical performance, expert raters recognise context as an important factor modulating their assessment of, for instance, trainee performance [46], [47] and [48] and take this along implicitly. Efforts should be made to make this process explicit. However, to assess the generalisability of our findings, more robust research needs to be done.

Although we chose optimal rater consistency by having the same rater for both the first and second rating of communication performance, the inherent limitation is that this may have caused a bias to corroborate the hypothesised findings. To minimise bias, the time lapse between both ratings was over one year. However, keeping in mind the explorative nature of the study, the results should be interpreted with care.

\subsection{Conclusion}

In conclusion, we did find indications that significant changes in communication performance scores can be found when assessing context specifically. In consultations where context factors are present that may strongly influence item scores, context-specific assessment may lead to different outcomes - also in GP trainee consultations. Therefore, we think that taking context factors into account may lead to a more valid assessment of communication performance.

\subsection{Practice implications}

\subsubsection{Implications for further research}


Essers, G., Dulmen, S. van, Es, J. van, Weel, C. van, Vleuten, C. van der, Kramer, A. Context factors in consultations of general practitioner trainees and their impact on communication assessment in the authentic setting. Patient Education and Counseling: 2013, 93(3), 567-572

In this study we found several context factors present in GP trainee consultations that may affect communication performance. Explicitly accounting for context factors in workplace-based communication assessment will not only make performance scores more transparent, it may also raise their external validity. We have made the process to incorporate context factors explicit and therefore open to empirical research. The rating process using the context-specific protocol may make a difference in GP trainee consultations but it also encompasses a long list of items to 'tick'. However, as communication is a context-dependent and goal-oriented activity [22], assessment can only be done properly if both are taken into account. Therefore, in future research, GP trainees could be asked to fill out the context factors checklist for each consultation. The hypothesis that context factors make a difference for performance scores can then be tested by rating these consultations twice: one by raters following the standard, generic way described in the MAAS-Global Manual and without any knowledge of the contextual factors and one by other raters that use the contextspecific rating protocol.

\subsubsection{Implications for education}

In the GP Specialty Training, the workplace experiences of GP trainees need to be taken more seriously. Knowing that they encounter the same context factors in their consultations, we need to help trainees use their knowledge of various context factors systematically in their communication and not only teach them to communicate 'by the book'. It would be useful to teach trainees to apply communication skills more deliberately attuned to patient-related and consultation-related context factors. Trainees can be made aware that applying communication skills is not a matter of 'one size fits all'. Professional communication requires adaptation to the specific characteristics of the patient and the situation. In training programmes, different contexts should explicitly be addressed. Explicating context factors presented in this paper can also help (oftentimes 'unconsciously competent') GP trainers to give adequate and specific feedback to their trainees.

Furthermore, in training communication assessment, raters can be made aware of the influence of context factors on communication and instructed to account for these factors in a protocolised way.

\section{COMPETING INTERESTS}

The study was funded by the SBOH Foundation, employer of Dutch General Practitioner Trainees and funder of the National Specialty Training for General Practitioners. There are no competing interests.

\section{ACKNOWLEDGEMENTS}

We thank the researchers at AMC Primary Care Department for generously granting access to the GP trainee consultations and the Dutch Ministry of Health for funding the collection and videotaping of routine GP consultations. Special thanks go to Boukje Andriesse who scored the presence of context factors in all the GP trainee consultations and to Tom Easten for his suggestions for editing the English writing. 
Essers, G., Dulmen, S. van, Es, J. van, Weel, C. van, Vleuten, C. van der, Kramer, A. Context factors in consultations of general practitioner trainees and their impact on communication assessment in the authentic setting. Patient Education and Counseling: 2013, 93(3), 567-572

\section{REFERENCES}

[1] S. Billett Constituting the workplace curriculum J Curric Stud, 38 (2006), pp. 31-48

[2] M.J. Ho, G. Yao, K.L. Lee, T.J. Hwang, M.C. Beach Long-term effectiveness of patientcentered training in cultural competence: what is retained? What is Lost? Acad Med, 85 (2010), pp. 660-664

[3] A.W.M. Kramer, H. Dusman, L.H.C. Tan, J.J.M. Jansen, R. Grol, C.P.M. van der Vleuten Acquisition of communication skills in postgraduate training for general practice Med Educ, 38 (2004), pp. 158-167

[4] C. Fernandez-Olano, J. Montoya-Fernandez, A.S. Salinas-Sanchez Impact of clinical interview training on the empathy level of medical students and medical residents Med Teach, 30 (2008), pp. 322-324

[5] S. Cheraghi-Sohi, P. Bower Can the feedback of patient assessments, brief training, or their combination, improve the interpersonal skills of primary care physicians? A systematic review BMC Health Serv Res, 8 (2008), p. 179

[6] M.E. Reinders, A.H. Blankenstein, H.E. van der Horst, D.L. Knol, P.L. Schoonheim, H.W.J. van Marwijk Does patient feedback improve the consultation skills of general practice trainees? A controlled trial Med Educ, 44 (2010), pp. 156-164

[7] Es. J. van, M. De Waard-Wieringa, M. Visser Differential growth in doctor-patient communication skills in GP trainees Med Educ, 47 (2013), pp. 691-700

[8] R.F. Brown, C.L. Bylund Communication skills training: describing a new conceptual model Acad Med, 83 (2008), pp. 37-44

[9] C. Heaven, J. Clegg, P. Maguire Transfer of communication skills training from workshop to workpace: the impact of clinical supervision Patient Educ Couns, 60 (2006), pp. 313-325

[10] J. Bensing, S. van Dulmen, K. Tates Communication in context: new directions in communication research Patient Educ Couns, 50 (2003), pp. 27-32

[11] R.L. Hulsman Shifting goals in medical communication. Determinants of goal detection and response formation Patient Educ Couns, 74 (2009), pp. 302-308

[12] S. Wilkinson, R. Perry, K. Blanchard, L. Linsell Effectiveness of a three-day communication skills course in changing nurses' communication skills with cancer/palliative care patients: a randomised controlled trial Pall Med, 22 (2008), pp. 365-375

[13] S. Hobma, P. Ram, A. Muijtjens, C. van der Vleuten, R. Grol Effective improvement of doctor-patient communication: a randomised controlled trial Brit J Gen Pract, 56 (2006), pp. 580-586

[14] A. Jerant, R.L. Kravitz, R. Azari, L. White, J.A. Garcia, H. Vierra et al. Training residents to employ self-efficacy-enhancing interviewing techniques: randomized controlled trial of a standardized patient intervention J Gen Intern Med, 24 (2009), pp. 606-613

[15] E.G. Price-Haywood, K.G. Roth, K. Shelby, L.A. Cooper Cancer risk communication with low health literacy patients: a continuing medical education program J Gen Intern Med, 25 (2010), pp. S126-S129

[16] D.M. Brock, L.B. Mauksch, S. Witteborn, J. Hummel, P. Nagasawa, L.S. Robins Effectiveness of intensive physician training in upfront agenda setting J Gen Intern Med, 26 (2011), pp. 1317-1323

[17] K.W. Eva On the generality of specificity Med Educ, 37 (2003), pp. 587-588

[18] W. Veldhuijzen, P.M. Ram, T. van der Weijden, S. Niemantsverdriet, C.P.M. van Der Vleuten Characteristics of communication guidelines that facilitate or impede guideline use: a focus group study BMC Fam Pract, 8 (2007), p. 31 
Essers, G., Dulmen, S. van, Es, J. van, Weel, C. van, Vleuten, C. van der, Kramer, A. Context factors in consultations of general practitioner trainees and their impact on communication assessment in the authentic setting. Patient Education and Counseling: 2013, 93(3), 567-572

[19] P. Salmon, B. Young Creativity in clinical communication: from communication skills to skilled communication Med Educ, 45 (2011), pp. 217-226

[20] J.M. Schirmer, L. Mauksch, F. Lang, M.K. Marvel, K. Zoppi, R.M. Epstein et al. Assessing communication competence: a review of current tools Fam Med, 37 (2005), pp. 184-192

[21] K. Zoppi, R.M. Epstein Is communication a skill? Communication behaviors and being in relation Fam Med, 34 (2002), pp. 319-324

[22] W. Veldhuijzen Challenging the patient-centred paradigm Maastricht University, Maastricht (2011)

[23] H. de Haes, J. Bensing Endpoints in medical communication research, proposing a framework of functions and outcomes Patient Educ Couns, 74 (2009), pp. 287-294

[24] D. Feldman-Stewart, M.D. Brundage, C. Tishelman, S.C. Team A conceptual framework for patient-professional communication: an application to the cancer context Psychooncology, 14 (2005), pp. 801-809

[25] L.A. Baig, C. Violato, R.A. Crutcher Assessing clinical communication skills in physicians: are the skills context specific or generalizable BMC Med Educ, 9 (2009), p. 22

[26] H.R. Winefield, T.G. Murrell, J.V. Clifford, E.A. Farmer The usefulness of distinguishing different types of general practice consultation, or are needed skills always the same? Fam Pract, 12 (1995), pp. 402-407

[27] P. Salmon, N. Mendick, B. Young Integrative qualitative communication analysis of consultation and patient and practitioner perspectives: towards a theory of authentic caring in clinical relationships Patient Educ Couns, 82 (2011), pp. 448-454

[28] J. Allen, B. Gay, H. Crebolder, J. Heyrman, I. Svab, P. Ram et al. The European definitions of the key features of the discipline and core competencies Brit J Gen Pract, 52 (2002), pp. 526-527

[29] D.J. Cegala, S.L. Broz Physician communication skills training: a review of theoretical backgrounds, objectives and skills Med Educ, 36 (2002), pp. 1004-1016

[30] G. Essers, A. Kramer, B. Andriesse, C. van Weel, C.P.M. van der Vleuten, A.M. van Dulmen Context factors in family physician communication - patient encounters and their impact on assessing communication skills - an exploratory study BMC Fam Pract, 14 (2013), p. 65

[31] J. de Jong, M.R.M. Maser, J. Mohrs, M. Wieringa-de Waard Opening the black box: the patient mix of GP trainees Brit J Gen Pract, 61 (2011), pp. e650-e657

[32] A. Bonney, L. Phillipson, S. Reis, S.C. Jones, D. Iverson Patients' attitudes to general practice registrars: a review of the literature Educ Prim Care, 20 (2009), pp. 371-378

[33] G. Essers, S. van Dulmen, C. van Weel, C. van der Vleuten, A. Kramer Identifying context factors explaining physician's low performance in communication assessment: an explorative study in general practice BMC Fam Pract, 12 (2011), p. 138

[34] J. van Es Tailoring to educational needs Academic Medical Centre, University of Amsterdam, Amsterdam (2012)

[35] J. van Thiel, H.F. Kraan, C.P. Van Der Vleuten Reliability and feasibility of measuring medical interviewing skills: the revised Maastricht History-Taking and Advice Checklist Med Educ, 25 (1991), pp. 224-229

[36] M.E. Reinders, A.H. Blankenstein, H.W.J. van Marwijk, D.L. Knol, P. Ram, H.E. van der Horst et al. Reliability of consultation skills assessments using standardised versus real patients Med Educ, 45 (2011), pp. 578-584

[37] P. Ram, R. Grol, J.J. Rethans, B. Schouten, C. van der Vleuten, A. Kester Assessment of general practitioners by video observation of communicative and medical performance in daily practice: issues of validity, reliability and feasibility Med Educ, 33 (1999), pp. 447-454 
Essers, G., Dulmen, S. van, Es, J. van, Weel, C. van, Vleuten, C. van der, Kramer, A. Context factors in consultations of general practitioner trainees and their impact on communication assessment in the authentic setting. Patient Education and Counseling: 2013, 93(3), 567-57

[38] W. Veldhuijzen, P.M. Ram, T. van der Weijden, M.R. Wassink, C.P.M. van der Vleuten Much variety and little evidence: a description of guidelines for doctor-patient communication Med Educ, 41 (2007), pp. 138-145

[39] A.A.R. Timmerman, P. Cals, J.W.L. Competentcy-based education of doctor-patient communication in Dutch General Practitioner specialty training Tijdschrift voor Medisch Onderwijs, 30 (2011), pp. 130-142

[40] S.O. Hobma, P.M. Ram, A.M.M. Muijtjens, R. Grol, C.P.M. van der Vleuten Setting a standard for performance assessment of doctor-patient communication in general practice Med Educ, 38 (2004), pp. 1244-1252

[41] M. Hojat, G. Xu A visitor's guide to effect sizes - statistical significance versus practical (clinical) importance of research findings Adv Health Sci Educ, 9 (2004), pp. 241-249

[42] W.P. Dunlap, J.M. Cortina, J.B. Vaslow, M.J. Burke Meta-analysis of experiments with matched groups or repeated measures designs Psychol Methods, 1 (1996), pp. 170-177 [43] J. Noordman, P. Verhaak, I. van Beljouw, S. van Dulmen Consulting room computers and their effect on general practitioner-patient communication Fam Pract, 27 (2010), pp. 644-651

[44] M. Jager, J. Schuling, J. Pols, J. Berents Differences in communication skills between trainee and experienced GPs Huisarts Wet, 54 (2011), p. 4

[45] P.P. Hobus, H.G. Schmidt, H.P. Boshuizen, V.L. Patel Contextual factors in the activation of first diagnostic hypotheses: expert-novice differences Med Educ, 21 (1987), pp. $471-476$

[46] B. Young, J. Ward, M. Forsey, K. Gravenhorst, P. Salmon Examining the validity of the unitary theory of clinical relationships: comparison of observed and experienced parentdoctor interaction Patient Educ Couns, 85 (2011), pp. 60-67

[47] S. Durning, A.R. Artino Jr., L. Pangaro, C.P. van der Vleuten, L. Schuwirth Context and clinical reasoning: understanding the perspective of the expert's voice Med Educ, 45

(2011), pp. 927-938

[48] M.J.B. Govaerts, L.W.T. Schuwirth, C.P.M. Van der Vleuten, A.M.M. Muijtjens Workplace-based assessment: effects of rater expertise Adv Health Sci Educ, 16 (2011), pp. 151-165 
Essers, G., Dulmen, S. van, Es, J. van, Weel, C. van, Vleuten, C. van der, Kramer, A. Context factors in consultations of general practitioner trainees and their impact on communication assessment in the authentic setting. Patient Education and Counseling: 2013, 93(3), 567-572

\section{FIGURE AND TABLES}

Fig. 1. Procedure of incorporating context factors into communication Sample of 44 consultations selected Standard rating with MAAS-Global (GE) assessment.

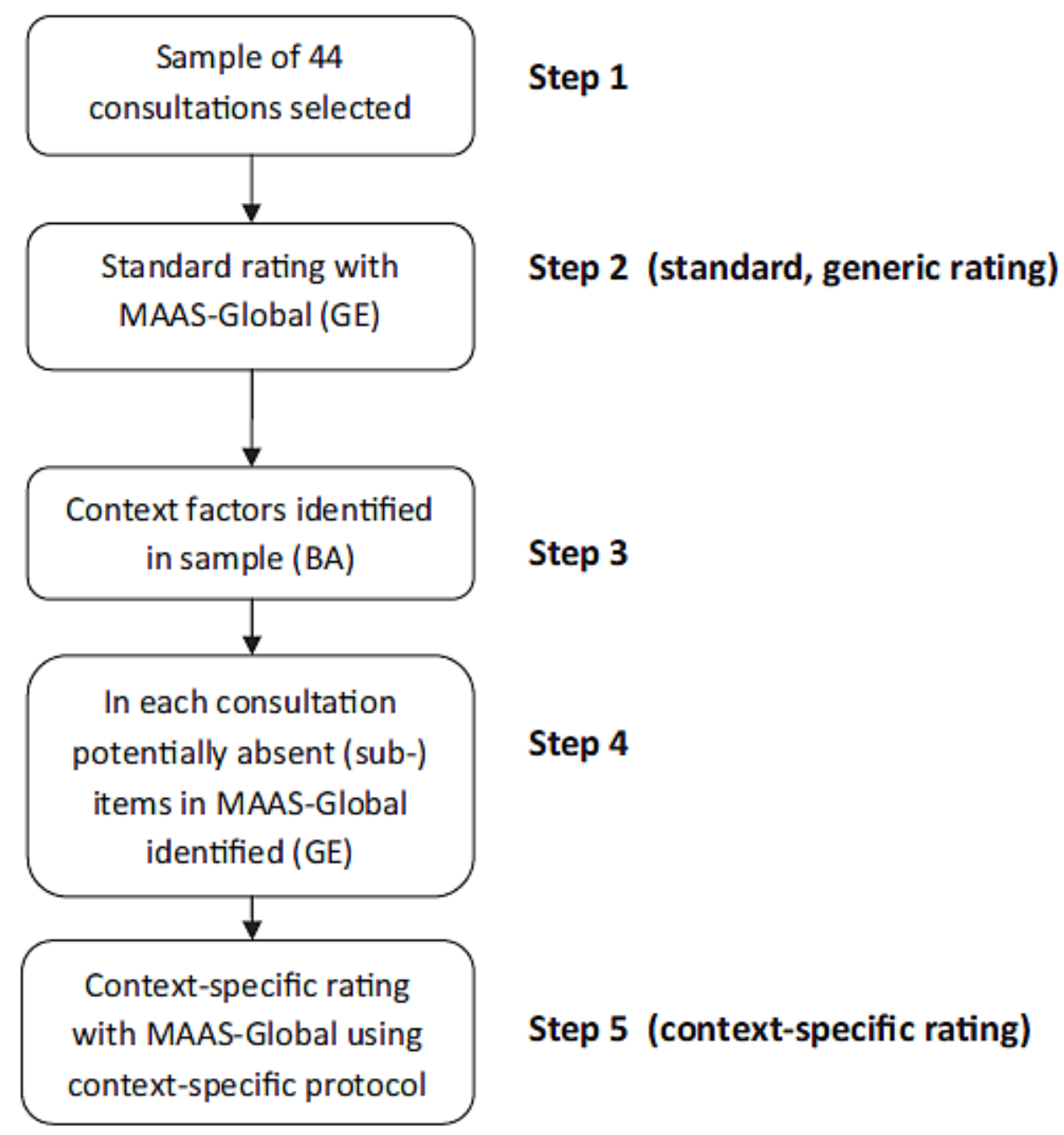


Essers, G., Dulmen, S. van, Es, J. van, Weel, C. van, Vleuten, C. van der, Kramer, A. Context factors in consultations of general practitioner trainees and their impact on communication assessment in the authentic setting. Patient Education and Counseling: 2013, 93(3), 567-572

Table 1

Context factors in $\mathrm{GP}^{\mathrm{a}}(N=40)$ vs. GP trainee $(N=44)$ consultations.

\begin{tabular}{|c|c|c|c|c|}
\hline & \multicolumn{2}{|c|}{ In GP } & \multicolumn{2}{|c|}{ In GP trainee } \\
\hline & $N$ & (\%) & $N$ & (\%) \\
\hline \multicolumn{5}{|l|}{ Doctor-related factors } \\
\hline 1. Doctor knows patient and his social context & 29 & (72) & 11 & (25) \\
\hline 2. Doctor knows patients' medical history & 35 & (88) & 21 & (48) \\
\hline 3. Doctor knows patients' way of communicating & 29 & (72) & 12 & (27) \\
\hline 4. Doctor is very experienced & 34 & (85) & 4 & (9) \\
\hline Doctor-related factors (total) & 127 & & 48 & \\
\hline \multicolumn{5}{|l|}{ Patient-related factors } \\
\hline 5. Specific patient verbal behaviour & 11 & (27) & 11 & (25) \\
\hline 6. Specific patient non-verbal behaviour & 1 & (3) & 9 & (20) \\
\hline 7. Patient is also treated by other provider & 12 & (30) & 14 & (32) \\
\hline 8. Patient has a disease (diagnosis) or (recurrent) problem known to both doctor and patient & 24 & (60) & 27 & (61) \\
\hline 9. Patient is familiar with (physical) examination (PE) & 23 & (58) & 28 & (64) \\
\hline Patient-related factors (total) & 71 & & 90 & \\
\hline \multicolumn{5}{|l|}{ Consultation-related factors } \\
\hline 10. Single consultation & 23 & (58) & 26 & (61) \\
\hline 11. First consultation in a series ${ }^{\mathrm{b}}$ & 4 & (10) & 10 & (23) \\
\hline 12. Follow-up consultation in a series ${ }^{c}$ & 11 & (28) & 9 & (20) \\
\hline 13. Consultation in a series based on protocol (initiative by doctor) ${ }^{d}$ & 1 & (3) & 1 & (2) \\
\hline 14. Consultation in preventive care (initiative doctor) ${ }^{\mathrm{e}}$ & 1 & (3) & 0 & (0) \\
\hline 15. Diagnosed problem is easily solved & 3 & (8) & 1 & (2) \\
\hline 16. Problem urgently needs medical care & 1 & (3) & 1 & (2) \\
\hline 17. Diagnosed problem is mainly psychosocial & 2 & (5) & 2 & (4) \\
\hline 18. There is more than one person (patient) present & 12 & (30) & 8 & (18) \\
\hline Consultation-related factors (total) & 57 & & 58 & \\
\hline \multicolumn{5}{|l|}{ 19. Characteristics of physical examination } \\
\hline -Simple & 30 & (75) & 26 & (59) \\
\hline -Invasive & 4 & (10) & 13 & (29) \\
\hline -Intimate & 6 & (15) & 16 & (36) \\
\hline -Superficial & 29 & (73) & 24 & (54) \\
\hline -Leaves room for talk & 10 & (25) & 9 & (20) \\
\hline -Requires full attention & 27 & $(68)$ & 36 & $(82)$ \\
\hline
\end{tabular}

a Data for GPs were drawn from Ref. [30].

${ }^{b}$ A first consultation in a series is a consultation in which no diagnosis is being made and the patient is referred for further tests, or in which doctor and patient agree to a follow-up consultation for check-up purposes.

c A follow-up consultation in a series is a second or consecutive consultation concerning a complaint or problem for which a referral was made, or that was agreed upon by patient and doctor. It is also marked as a follow-up consultation if the patient has been referred for specialist care (even if surgery took place) and the patient comes back to report on that (no maximum time span is indicated).

d A protocol based consultation is a consultation, concerning a prior diagnosed complaint or problem, that is initiated by the doctor on the basis of a protocol or clinical practice guideline.

e A preventive consultation is a consultation that is initiated by the doctor on the basis of a protocol or clinical practice guideline (diagnosis or disease is not necessarily established). 
Essers, G., Dulmen, S. van, Es, J. van, Weel, C. van, Vleuten, C. van der, Kramer, A. Context factors in consultations of general practitioner trainees and their impact on communication assessment in the authentic setting. Patient Education and Counseling: 2013, 93(3), 567-572

nive

Table 2

Results of application of context-specific rating protocol GP trainee vs. GP consultations.

\begin{tabular}{|c|c|c|c|c|c|c|c|}
\hline \multirow[t]{2}{*}{ MAAS-Global item } & \multirow{2}{*}{$\begin{array}{l}\text { GP trainee } \\
\text { Score without } \\
\text { context } \\
\text { factors (SD) }\end{array}$} & \multirow{2}{*}{$\begin{array}{l}\text { GP } \\
\text { Score without } \\
\text { context } \\
\text { factors (SD) }\end{array}$} & \multirow{2}{*}{$\begin{array}{l}\text { GP trainee } \\
\text { Score with } \\
\text { context } \\
\text { factors (SD) }\end{array}$} & \multirow{2}{*}{$\begin{array}{l}\text { GP } \\
\text { Score with } \\
\text { context } \\
\text { factors (SD) }\end{array}$} & \multirow[t]{2}{*}{$\begin{array}{l}\text { Minimum } \\
\text { standard } \\
\text { score }^{\text {b }}\end{array}$} & \multirow{2}{*}{$\begin{array}{l}\text { GP trainee } \\
\text { Effect size }\end{array}$} & \multirow{2}{*}{$\begin{array}{l}\text { GP } \\
\text { Effect size }\end{array}$} \\
\hline & & & & & & & \\
\hline Introduction ${ }^{* * G P}$ trainee, ${ }^{*} G P$ & $3.39(0.94)$ & $3.60(0.93)$ & $4.00(0.83)$ & $4.10(0.90)$ & 4.0 & 0.67 & 0.55 \\
\hline Follow-up consultation & $3.00(1.05)$ & $3.83(0.58)$ & $3.30(1.06)$ & $4.42(0.79)$ & - & 0.22 & 0.87 \\
\hline Request for help “'GP & $2.68(1.50)$ & $1.00(1.45)$ & $2.93(1.86)$ & $1.67(2.23)$ & 3.1 & 0.15 & 0.32 \\
\hline Physical Examination ${ }^{\cdots G P}$ trainee and GP & $4.12(0.89)$ & $4.33(1.51)$ & $5.38(0.88)$ & $5.14(1.31)$ & 3.6 & 1.42 & 0.57 \\
\hline Diagnosis ${ }^{*}$ "GP & $4.09(0.98)$ & $3.84(0.69)$ & $4.34(0.86)$ & $4.46(0.80)$ & 3.7 & 0.17 & 0.80 \\
\hline Management ${ }^{\circ}$ GP trainee & $2.81(1.24)$ & $2.90(1.15)$ & $3.20(1.21)$ & $3.25(1.15)$ & 3.4 & 0.31 & 0.30 \\
\hline Closure ${ }^{\circ \mathrm{GP}}$ & $0.66(1.21)$ & $2.62(0.92)$ & $0.66(1.07)$ & $2.37(2.19)$ & 2.1 & 0.00 & 0.15 \\
\hline Exploration “.GP & $2.23(1.24)$ & $1.85(1.49)$ & $2.52(1.37)$ & $2.57(1.53)$ & 3.0 & 0.23 & 0.48 \\
\hline Emotions ${ }^{* * \mathrm{GP}}$ & $1.17(1.46)$ & $0.88(1.27)$ & $1.32(1.57)$ & $1.60(1.94)$ & 2.3 & 0.10 & 0.44 \\
\hline Information giving " $\mathrm{GP}$ & $3.77(0.80)$ & $3.67(0.97)$ & $4.00(0.87)$ & $4.07(1.05)$ & 3.2 & 0.29 & 0.40 \\
\hline Summarising ${ }^{\cdots G P}$ trainee and GP & $3.93(1.62)$ & $3.27(1.28)$ & $4.50(1.55)$ & $4.00(1.97)$ & 2.6 & 0.36 & 0.42 \\
\hline Structuring ${ }^{\circ}$ GP trainee and GP & $4.18(1.32)$ & $3.85(1.21)$ & $4.77(1.00)$ & $4.80(1.36)$ & 3.6 & 0.50 & 0.77 \\
\hline Empathy ${ }^{\cdots G \mathrm{GP}}$ & $4.32(1.09)$ & $4.25(0.90)$ & $4.52(1.07)$ & $4.87(1.28)$ & 3.5 & 0.18 & 0.56 \\
\hline Total mean score ${ }^{\cdots G P}$ trainee and GP & $3.09(0.70)$ & $2.98(0.61)$ & $3.51(0.75)$ & $3.66(0.98)$ & 3.4 & 0.57 & 0.84 \\
\hline
\end{tabular}

a Data for GPs were drawn from Ref. [30].

b Minimum standard score set by Ref. [40].

$p<.05$.

. $p<.01$.

$p<.000$. 